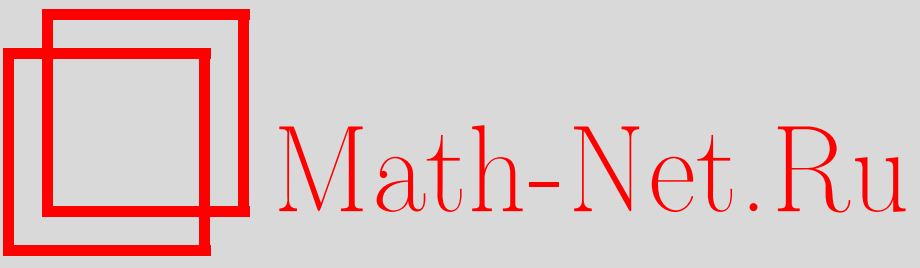

С. Ю. Оревков, Новая аффинная $M$-секстика. II, УMH, 1998, том 53, выпуск 5, 243-244

DOI: https://doi.org/10.4213/rm83

Использование Общероссийского математического портала Math-Net.Ru подразумевает, что вы прочитали и согласны с пользовательским соглашением

http://www . mathnet.ru/rus/agreement

Параметры загрузки:

IP: 18.208 .226 .222

26 апреля 2023 г., 09:22:10 


\section{НОВАЯ АФФИННАЯ $M$-СЕКСТИКА. II}

\section{С. Ю. ОревКОВ}

Аффинная вещественная алгебраическая кривая назьвается аффинной $M$-кривой если она имеет максимально возможное разрешенное неравенством Харнака число компонент связности, в частности, афффинная $M$-секстика имеет 16 компонент связности, 10 из которых являются овалами, и осталшные 6 получены из 11-го овала проективизации выкидьванием бесконечно удаленных точек. В настоящей заметке мы продолжаем изотопическую классификацию аффинных $M$-секстик, начатую в [1]-[5], и приводим подробности реализации изотопического типа $A_{3}(0,5,5)$ (см. рис. 1 , где $L$ - бесконечно удаленная прямая). Он был ошибочно объявлен запрещенным в [3]. Результат и идея доказательства были анонсированы в [5, §2].

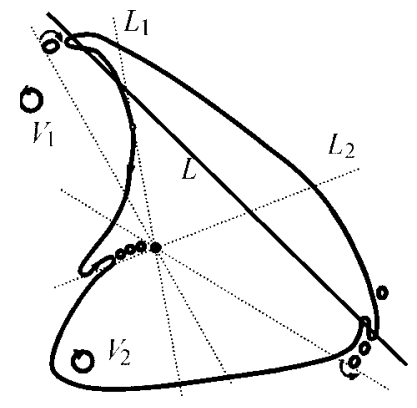

Рис. 1

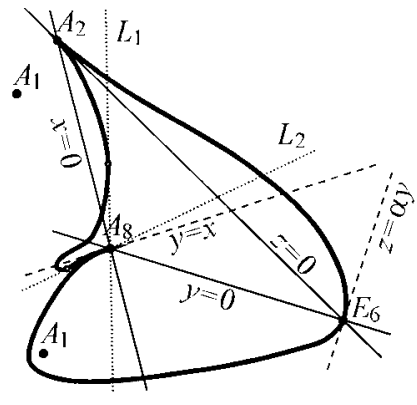

Рис. 2

Построение похоже на реализацию $B_{2}(1,8,1)$, приведенную в [4]. Однако, мы здесь используем геометрические рассуждения, позволяющие сократить вьгисления. Такими же рассуждениями можно упростить доказательство в [4].

Построим плоскую рациональную секстику $C$ с особенностями $E_{6}, A_{8}, A_{2}, A_{1}, A_{1}$, такую, что прямая, проходящая через $E_{6}$ и $A_{2}$ (обозначим ее через $L$ ), касается $C$ в $A_{2}$. Выберем координаты $(x: y: z)$ как на рис. 2 и рассмотрим параметризацию $t \mapsto(x: y: z)$ кривой $C$, такую что $0 \mapsto A_{8}, 1 \mapsto A_{2}, \infty \mapsto E_{6}$. Тогда

$$
x(t)=t^{2}\left(b t^{2}+c t+1\right)(t-1)^{2}, \quad y(t)=t^{2}(a t+1), \quad z(t)=(t-1)^{3} .
$$

Особенности $E_{6}$ и $A_{2}$ обеспечены (1). Условие на $A_{8}$ имеет вид

$$
\exists \gamma_{2}, \gamma_{3} \text { такие, что } \quad \operatorname{ord}_{t=0}\left(v z^{2}-\gamma_{2} u^{2} z-\gamma_{3} u^{3}\right)=8,
$$

где $u=x+y, v=x-y$. Раскладывая $v z^{2}-\gamma_{2} u^{2} z-\gamma_{3} u^{3}$ в виде $e_{3} t^{3}+e_{4} t^{4}+\ldots$ где $e_{j}$ - полиномиальные выражения от переменных $a, b, c, \gamma_{2}, \gamma_{3}$, мы видим, что (2) дает систему уравнений и неравенств $e_{3}=\cdots=e_{7}=0, e_{8} \neq 0$. Эта система имеет 4 решения, два из которых вешественны. Мы выберем решение $a=\alpha, b=-\left(6 \alpha^{2}+11 \alpha+3\right) / 11, c=\alpha+2$, $\gamma_{2}=\left(6 \alpha^{2}+33 \alpha+36\right) / 44, \gamma_{3}=-\left(111 \alpha^{2}+374 \alpha+347\right) / 968$, где $\alpha=-5.1046 \ldots$ - единственный вещественный корень уравнения

$$
3 \alpha^{3}+24 \alpha^{2}+51 \alpha+34=0
$$

Лемма. С расположена относительно координатных осей как на рис. 2.

СледСтвиЕ. (см. Лемму в [6].) С можно сгладить так, как изображено на рис. 1.

Работа выполнена при частичной финансовой поддержке грантов РФФИ-96-01-01218 и DGICYT SAB95-0502. 
Лемму можно доказать методом работы [4], но это требует компьютерных вычислений. Здесь мы даем геометрическое доказательство, в котором все можно проверить вручную. Корни (с учетом кратностей) многочленов $x(t), y(t), z(t)$ таковы:

$$
\begin{array}{ccccccc}
x(t): & -0.53 \ldots & 0 & 0 & 0.20 \ldots & 1 & 1 \\
y(t): & 0 & 0 & 1.19 \ldots & \infty & \infty & \infty
\end{array}
$$

Значит, $C$ пересекает координатные оси в порядке, показанном на рис. 2 . Посколшку $\gamma_{2} \approx 0.543>$ 0 , ветвь кривой $C$ в $A_{8}$ расположена относительно касательной $x=y$ как на рис. 2 . Из формулы рода следует, что у $C$ есть еще две точки $A_{1}$. Они вещественны, так как иначе мы получили бы противоречие с правилом чередования ориентаций [7] на отрезке пучка прямых $\left[L_{1} L_{2}\right]\left(\mathrm{cm}_{\text {. }}\right.$ рис. 2). Покажем, что каждая из точек $A_{1}$ дает при надлежащем сглаживании пустой овал (овал называется пустьм, если внутри него нет других овалов). Координатные оси делят $C_{0}$ на 6 дуг. Три из них не могут пересекать осталшные, так как они разделены координатными осями. Мы имеем $y(t)-\alpha z(t)=(3 \alpha+1) t^{2}-3 \alpha t+\alpha$. Дискриминант равен $-3 \alpha^{2}-4 \alpha \approx-57.7<0$, значит, касательная $y=\alpha z$ в точке $E_{6}$ разделяет все остальные пары дуг кроме тех двух дуг, которые выходят из $A_{8}$. Если они пересекались бы в двух точках, то существовало бы $M$-сглаживание с 6 внутренними овалами, что невозможно. Если же они пересекались бы в одной точке, то три внутренних овала были бы отрицательными, что противоречит формуле Рохлина для комплексных ориентаций [8]. Таким образом, каждая из точек $A_{1}$ является либо уединенной точкой, либо точкой самопересечения одной из дуг. В любом случае есть сглаживание, дающее пустой овал. Как мы показали вьше, одна из точек $A_{1}$ должна быть между $L_{1}$ и $L_{2}$. Обозначим через $V_{2}$ овал, полученньй ее сглаживанием. Те же рассуждения применительно к отрезку $-\infty<\lambda<0$ пучка прямых $y=\lambda x$ (возмущения прямых $x=0$ и $y=0$, а также некоторые комплексные ориентации изображены на рис. 1) показьвают, что другая точка $A_{1}$ (обозначим соответствующий овал через $\left.V_{1}\right)$ должна лежать в полуплоскости $H=\{x y>0\}$. Предположим, что овал $V_{1}-$ внутренний. Тогда он не может лежать в левом секторе множества $H$ (по теореме Безу), но если он лежал бы в правом секторе, то он был бы ориентирован по часовой стрелке, что опять противоречит правилу чередования ориентаций.

Таким образом, овал $V_{1}-$ внешний, значит, $V_{2}$ - внутренний, так как число внутренних овалов $M$-секстики не может равняться 4 . Из формулы комплексных ориентаций следует, что $V_{2}$ ориентирован по часовой стрелке, значит, по правилу чередования ориентаций, $V_{2}$ лежит в левом нижнем секторе множества $\mathbf{R P}^{2} \backslash\left(L_{1} \cup L_{2}\right)$. Из компексных ориентаций видно (см. рис. 1$)$, что обе точки $A_{1}$ - уединенные. Лемма доказана.

ЗАмЕчАниЕ. В работе [6] приведено доказательство существования независимого сглаживания особенностей только в случае неприводимой проективной кривой. Однако, аналогичньй резулштат для приводимых кривых доказывается точно также (см. замечание в конце [6]). Наше построение можно свести к неприводимому случаю. Для этого достаточно явно выписать деформацию кривой $C$, положив $z(t)=(t-1)^{3}(1-\varepsilon t)$ в $(1)$ и сохранив условие $(2)$.

\section{СПИСОК ЛИТЕРАТУРЫ}

[1] Полотовский Г. М. (М - 2)-кривые 8-го порядка: построения, открытые вопросы // Депонировано в ВИНИТИ, № 1185-85Деп 1984. С. 1-195. [2] Корчагин А. Б., Шустин Е. И. // Изв. АН СССР. Сер. матем. 1988. Т. 52. №6. С. 1181-1199. [3] Шустин Е. И. // Методы качественной теории и теории бифуркаций. Горький: Изд-во ГГУ, 1988. С. 97-105. [4] Оревков С. Ю. // Функц. анализ и его прилож. 1998. Т. 32. № 2. С. 91-94. [5] Orevkov S. Yu. Link theory and oval arrangements of real algebraic curves // Topology. (to appear). [6] Шycтин Е. И. // Матем. заметки. 1987. Т. 42. № 2. С. 180-186. [7] Фидлер Т. // Изв. АН СССР. Сер. матем. 1982. Т. 46. С. 853-863. [8] Рохлин В. А. // Функц. анализ и его прилож. 1974. Т. 8. № 4. C. $71-75$. 